\title{
Wenn Mitarbeiter als Konsumenten produktiv sind
}

\section{Zur Rolle des Mitarbeiterkonsums in entgrenzter Erwerbsarbeit}

\section{Von Viola Muster ${ }^{1}$}

Zusammenfassung: Vor dem Hintergrund verschiedener Entgrenzungsprozesse in Erwerbsarbeit und Konsum wird die Doppelrolle von Personen als Erwerbstätige und Konsumenten bislang kaum betrachtet. Der Artikel zeigt, dass Unternehmen jedoch längst die Doppelrolle ihrer Mitarbeiter als Konsumenten berücksichtigen. Dabei sind Mitarbeiter einerseits Adressaten von unternehmerischen Konsumangeboten und andererseits Akteure konsumbezogener Leistungserbringung. Es wird beleuchtet, dass dem Konsum von Mitarbeitern zentrale Funktionen für die Entgrenzung und Subjektivierung von Erwerbsarbeit zukommen. Deutlich wird, dass Entgrenzungsprozesse im Erwerbsarbeits- und im Konsumbereich nicht zwangsläufig getrennt voneinander ablaufen oder unterschiedliche Zielgruppen betreffen müssen, sondern auf Subjektebene zusammentreffen können.

\section{Einleitung}

Strukturelle Veränderungen in Konsum und Erwerbsarbeit haben dazu geführt, dass Konsumenten und Erwerbstätige zunehmend auf persönliche Ressourcen und private Bereiche zurückgreifen, um Konsum- und Erwerbsarbeitsleistungen zu erbringen. Konsumenten arbeiten an verschiedenen Stellen der Wertschöpfungskette in unterschiedlichen Konsumfeldern aktiv mit: Bankgeschäfte regeln sie allein vor dem Laptop, Schränke bauen sie eigenhändig zusammen und in Internetforen geben sie Unternehmen Tipps für Produktverbesserungen (u.a. Toffler 1980; Grün / Brunner 2002; Voß / Rieder 2005; Blättel-Mink / Hellmann 2010; Kleemann et al. 2012). Erwerbstätige sind flexibel und mobil. Sie erledigen Aufgaben von zu Hause aus, sind ständig erreichbar oder wechseln ihre Wohnorte für einen neuen Job (u.a. Voß 1998; Voß / Pongratz 1998; Kratzer 2003; Pongratz / Voß 2003; Gottschall / Voß 2005; Jurczyk et al. 2009).

Voß und Rieder (2005) konstatieren, dass hinter beiden Entwicklungssträngen ein ökonomisches Prinzip steht: der verbesserte Zugriff von Unternehmen auf eine erweiterte Ressourcenbasis von Personen. Sie sehen hinter beiden Entwicklungssträngen eine komplementäre Entgrenzungslogik, die Unternehmen der Optimierung von Betriebsabläufen und einer gesteigerten Wertschöpfung dient. Ihr Verweis auf die strukturelle Verbindung zwischen ,entgrenztem Konsum“ und „entgrenzter Erwerbsarbeit" stellt in Hinblick auf die sonst weitgehend voneinander getrennten Forschungsfelder eine Besonderheit dar. Auf Subjektebene wird jedoch auch von ihnen nicht beachtet, dass viele Menschen nicht entweder Erwerbsarbeitskraft oder Konsument sind, sondern meist beide Rollen miteinander vereinbaren (müssen). Sie agieren in einer Doppelrolle als „konsumierende Erwerbstätige“ und „,erwerbstätige Konsumenten“ im Alltag.

Auch wurde bislang kaum betrachtet, dass nicht nur Erwerbstätige oder Konsumenten private Fähigkeiten und Leistungen in Unternehmensprozesse einbringen, sondern auch Erwerbstätige als Konsumenten, also in ihrer Doppelrolle, in Unternehmen berücksichtigt werden und Leistungen erbringen. Einerseits werden Mitarbeiter ${ }^{2}$ als Konsumenten in Unternehmen adressiert, weil der Konsum ein zentrales Instrument darstellt, um die Leistungsbereitschaft und -fähigkeit der Mitarbeiter zu fördern. Unternehmerische Konsumangebote

1 Ich danke den anonymen Gutachtern, Vera Fricke und Ulf Schrader für ihre konstruktiven Hinweise.

2 Erwerbstätige, die Arbeiter oder Angestellte einer Organisation sind, werden im Folgenden als Mitarbeiter bezeichnet. 
werden im Erwerbsarbeitskontext bereitgestellt oder aber auch zur Nutzung im Privatleben ermöglicht. Andererseits tragen Mitarbeiter als Konsumenten direkt zur Wertschöpfung eines Unternehmens bei. Als kreative Konsumenten arbeiten Mitarbeiter in Innovationsprozessen mit (z.B. Schweisfurth 2013), als umweltorientierte Konsumenten bringen Mitarbeiter das Umwelt- und Nachhaltigkeitsmanagement voran (z.B. Schrader / Harrach 2013) und als authentische Konsumenten agieren Mitarbeiter als Markenbotschafter der Unternehmensprodukte (z.B. Henkel et al. 2007).

Ziel dieses Artikels ist es, die Doppelrolle von Personen als Mitarbeiter und Konsumenten in Unternehmen zu beleuchten. Dabei soll gezeigt werden, inwieweit eine Entgrenzung von Erwerbsarbeit unmittelbar mit Konsum verknüpft ist bzw. mittels Konsum vorangetrieben wird. Darüber hinaus soll gezeigt werden, dass entgrenzte Erwerbarbeit und entgrenzter Konsum nicht nur, wie von Voß und Rieder (2005) dargestellt, einen möglichen strukturellen Zusammenhang haben, sondern auch auf Subjektebene die Entgrenzungen in beiden Bereichen zusammentreffen können.

Zunächst wird im zweiten Kapitel zusammengefasst, was eine Entgrenzung von Erwerbsarbeit und eine Entgrenzung von Konsum kennzeichnet. Danach wird die Doppelrolle von Mitarbeitern als Konsumenten und der Zusammenhang zwischen dieser Doppelrolle und der Entgrenzung von Erwerbsarbeit näher beleuchtet: Im dritten Kapitel geht es dabei um Mitarbeiter als Adressaten von Konsumangeboten. Im vierten Kapitel stehen Mitarbeiter als Akteure konsumbezogener Leistungserbringung im Vordergrund. Im fünften Kapitel wird diskutiert, welche Chancen und Risiken diese Verknüpfung von Mitarbeiter- und Konsumentenrolle für die einzelnen Personen haben kann. Abschließend wird ein Fazit gezogen.

\section{Die doppelte Entgrenzung von Erwerbsarbeit und Konsum}

Im Folgenden werden die strukturellen Veränderungen in Erwerbsarbeit und Konsum, die darin liegenden Chancen und Risiken für Personen und die bestehenden Verbindungslinien zwischen beiden Forschungsbereichen skizziert.

\subsection{Entgrenzung und Subjektivierung von Erwerbsarbeit}

Die Auflösung und Verschiebung zeitlicher, räumlicher, organisatorischer und inhaltlicher Strukturierungen haben Erwerbsarbeit grundlegend verändert (u.a. Voß 1998; Voß / Pongratz 1998; Kratzer 2003; Moldaschl / Voß 2003; Pongratz / Voß 2003; Gottschall / Voß 2005). Indem mit Hilfe unterschiedlicher Führungs- und Arbeitsorganisationskonzepte (z.B. partizipativer Führung, Empowerment, Vertrauensarbeitszeit, Intrapreneurship, Projektarbeit etc.) feste Regelungen, Zuständigkeitsbereiche und Kontrollen in Unternehmen gelockert und abgebaut werden, entsteht ein „Freiraum“ für die Mitarbeiter, der die Entwicklung neuer Strukturierungen möglich und zugleich auch nötig macht.

Die Mitarbeiter dürfen und müssen nun gleichermaßen ihre Arbeit selbst organisieren, selbst gestalten und selbst kontrollieren (Voß 1998; Voß / Pongratz 1998; Kratzer 2003). Indem sie mehr „Selbst“ in ihre Erwerbsarbeit einbringen, werden Ressourcen und Kompetenzen freigelegt und für unternehmerische Wertschöpfungsprozesse verwertbar gemacht, die vor allem im Vergleich zu tayloristisch-fordistisch organisierten Produktionsprozessen eine bislang eher untergeordnete Rolle spielten. Dabei handelt es sich einerseits um subjektbezogene, also der Person eigene und durch die Lebenshistorie der Person individuell entwickelte Kompetenzen und Fähigkeiten, die zur Ausgestaltung der Arbeit, aber auch in der Koordination mit anderen oder im Umgang mit sich selbst nun von zunehmender Bedeutung sind (Kleemann / Matuschek / Voß 1999; Kratzer 2003; Lohr 2003; Kleemann 2012). Beispiele hierfür sind Kreativität, Innovativität, Eigenmotivation, Empathie, Selbstkontrolle oder 
Selbstdisziplin. Erwerbsarbeit wird in Folge dessen subjektiviert. Andererseits handelt es sich dabei um zeitlich-räumliche, materielle oder auch soziale Ressourcen, die aus privaten Bereichen genutzt werden (Voß / Pongratz 1998; Kratzer 2003). Beispiele dafür sind die Weiterarbeit zu Hause oder die Nutzung von privaten Kontakten für erwerbsarbeitsbezogene Leistungen.

Die mögliche und zugleich notwendige Integration von Subjektivität und privaten Ressourcen in Arbeitsprozesse ist nicht nur für Unternehmen chancenreich, da sie ihnen - quasi als kostengünstiger Ersatz für ihre entfallenen Strukturierungsleistungen - die Leistungserbringung garantiert und durch den Zugriff auf eine erweiterte Ressourcenbasis Leistungssteigerungen möglich macht. Auch für Mitarbeiter entstehen dabei zahlreiche Chancen. So erhalten sie dadurch die Möglichkeit, Erwerbsarbeit individueller und flexibler zu gestalten. Die auszufüllenden Freiräume können dabei den Individualisierungs- und Selbstverwirklichungsansprüchen, die Personen zunehmend an Erwerbsarbeit stellen, entgegenkommen (Kleemann / Matuschek / Voß 1999; Lohr 2003). Mehr Arbeitszufriedenheit, Sinnstiftung und subjektives Wohlbefinden können daraus folgen.

Der verstärkte Einbezug der ,ganzen Person“ ist jedoch - vor allem für die Mitarbeiter auch riskant. Die individuellen Kapazitäten zur Nutzung dieser Gestaltungschancen, aber auch zum Umgang mit den erhöhten Anforderungen sind schließlich nicht gleich verteilt (Voß / Pongratz 1998; Kleemann / Matuschek / Voß 1999). Mitarbeiter bringen nicht nur unterschiedliche Kompetenzen und Ressourcen aus ihrem Privatleben mit (die mehr oder weniger gut für die Leistungserbringung geeignet sind), sie verfügen auch über unterschiedliche Strategien und Möglichkeiten, diese Eigenschaften im Privatleben zu entwickeln und - fast noch wichtiger aufrechtzuerhalten und zu regenerieren (Jürgens 2006). Das kann, wenn die verfügbaren subjektiven Potentiale und privaten Ressourcen nicht zur Erfüllung der Erwerbsarbeitsanforderungen ausreichen, für Mitarbeiter mit Überforderung, permanenter Unsicherheit und Misserfolg einhergehen (Voß 1998; Voß / Pongratz 1998; Jürgens 2006; Moosbrugger 2012). Zudem kann daraus folgen, dass Mitarbeiter, die für sie notwendigen Schutzräume und Regenerationsmöglichkeiten im Privatleben nicht aufrechtzuerhalten in der Lage sind. Der private Alltag und schließlich der gesamte Lebenszusammenhang stehen dann zur Disposition, um den Erwerbsarbeitsanforderungen gerecht zu werden (Voß 1998; Voß / Pongratz 1998; Kleemann / Matuschek / Voß 1999; Kratzer 2003; Jürgens 2006).

\subsection{Entgrenzung von Konsum}

Die produktiven Leistungen, die in der Konsumsphäre erbracht werden, fanden in der Mainstream-Ökonomie lange Zeit kaum Beachtung. Schon immer müssen Marktprodukte oftmals (weiter-)verarbeitet, verändert oder kombiniert werden, damit sie überhaupt zur individuellen Bedürfnisbefriedigung beitragen (Scherhorn 1980; Schweitzer 1991). Scherhorn (1980: 103) schreibt dazu, dass ,,[e]in großer Teil der Aktivitäten, die die Bedürfnisse von Konsumenten befriedigen, im Konsumsektor mit dessen Mitteln selbst produziert" wird. Für diese privaten Produktionsleistungen benötigen Konsumenten verschiedene Ressourcen wie Wissen und Fähigkeiten (Kompetenzen), (Arbeits-)Kraft, Aufmerksamkeit oder Zeit. Immer dann, wenn diese privaten Ressourcen zum Einsatz kommen, sind Konsumenten produktiv.

Vor allem aus soziologischer und betriebswirtschaftlicher Sicht wurden in den letzten Jahren produktive Konsumleistungen unter den Schlagworten „Prosuming“ (Blättel-Mink / Hellmann 2010 mit Bezug auf Toffler 1980), Kundenarbeit (Voß / Rieder 2005), Ko-Produktion (Hanekop / Wittke 2008, 2012) bzw. Co-Produktion (Grün / Brunner 2002, 2003) oder Co-Creation (Prahalad / Ramaswamy 2000; Zwick / Bonsu / Darmody 2008) verstärkt thematisiert. Dabei geht es darum, dass Konsumenten in neuer Qualität und Quantität Aufgaben erbringen, die von Unternehmen angestoßen werden. 
Ein zentrales Aktionsfeld der sogenannten Kundenarbeit ist die Selbstbedienung (SB), die vormals angebotene Dienstleistungen ersetzt bzw. ergänzt (Voß / Rieder 2005). Während Toffler bereits 1980 auf diese seit den 60er Jahren bestehende Entwicklung, beispielsweise in Hinblick auf die Ausbreitung von SB-Supermärkten und SB-Baumärkten, aufmerksam gemacht hat, zeigt sich durch technische Innovationen, vor allem die Ausbreitung und Weiterentwicklung des Internets, nach wie vor eine große Veränderungsdynamik in diesem Feld (z.B. Online-Banking, Online-Shopping, Online-Booking etc.) (Voß / Rieder 2005).

Eine andere Art der Kundenarbeit stellt die Einbindung von Kunden in Unternehmensprozesse dar. Im Unterschied zur Selbstbedienung wird dabei nicht das „Ende“ einer Wertschöpfungskette oder die gesamte Leistung ausgelagert, sondern der Wertschöpfungsprozess an verschiedenen Stufen (z.B. Produktentwicklung, Produktdesign, Marketing, etc.) durch die Mithilfe des Kunden angereichert. Auch hier spielt das Internet eine große Rolle. So werden beim „Crowdsourcing“ Kunden dazu angeregt, mit den Unternehmen, beispielsweise in sozialen Netzwerken oder Foren, in Kontakt zu treten und ihnen Produktfeedback zu geben, bei Problemlösungen zu helfen oder Verbesserungsvorschläge mitzuteilen (Reichwald et al. 2007; Kleemann / Rieder / Voß 2008; Kleemann et al. 2012).

Unternehmen können durch Kundenarbeit effizienter werden und damit Kosten sparen (z.B. Ticketautomat statt Schalterbedienung), aber auch ihre Effektivität steigern (z.B. durch Produktinnovationen, die besser den Kundenwünschen entsprechen) (Voß / Rieder 2005; Reichwald et al. 2007). Konsumenten können von niedrigeren Preisen und einer höheren Flexibilität bei der Leistungsinanspruchnahme profitieren. Ihnen nutzen die Ergebnisse der Kundenarbeit - wenn auch teilweise, beispielsweise im Bereich des Crowdsourcing, eher indirekt und zeitverzögert - indem Produkte entstehen, die besser ihren Wünschen und Bedürfnissen entsprechen. Kundenarbeit kann Spaß machen, die Identifikation mit dem Produkt erhöhen, Genugtuung verschaffen und Selbstwirksamkeitserfahrungen befördern (Kleemann / Rieder / Voß 2008; Kleemann et al. 2012).

Die Übertragung von Leistung und Verantwortung auf den einzelnen Konsumenten bringt jedoch auch Risiken mit sich: Nicht alle Konsumenten sind in der Lage, die Anforderungen erfolgreicher - im Sinne von ihrer Bedürfnisbefriedigung zuträglicher - Kundenarbeit zu erfüllen (Voß / Rieder 2005). Den verringerten Kosten und der größeren Flexibilität bei der Selbstbedienung müssen beispielsweise ein erhöhter Zeit- und Kraftaufwand und ein selbst zu organisierender Wissens- und Kompetenzerwerb entgegengesetzt werden. Kunden, die diese Voraussetzungen nicht erfüllen (können), müssen dann unter Umständen auf diese Leistungen verzichten oder können sie nur mit Nachteilen in Bezug auf Qualität und Kosten in Anspruch nehmen.

\subsection{Verbindungslinien zwischen entgrenzter Erwerbsarbeit und entgrenztem Kon- sum}

Die strukturellen Veränderungen in der Erwerbsarbeit und im Konsum werden überwiegend von unterschiedlichen Forschungsdisziplinen untersucht und kaum miteinander in Verbindung gesetzt. Voß und Rieder (2005) stellen hierbei eine Ausnahme dar, da sie hinter beiden Entwicklungen eine gemeinsame Systemlogik erkennen. Unabhängig von dieser möglichen strukturellen Verknüpfung bleibt offen, wie Personen die Veränderungen in Erwerbsarbeit und Konsum in ihrer alltäglichen Lebensführung ${ }^{3}$ bewältigen. Während die individuellen Chancen und Risiken, wie zuvor dargestellt, in ihren Einzelbereichen diskutiert werden, bleibt bislang unklar, welche Chancen und Risiken sich für Personen ergeben, die im Alltag sowohl im Erwerbsarbeitsbereich als auch im Konsumbereich mit veränderten Handlungs-

3 Zum Konzept der alltäglichen Lebensführung siehe z.B. Voß 1991; Jurczyk / Rerrich 1993. 
anforderungen und -möglichkeiten umgehen und diese miteinander vereinbaren (können und müssen). Es kann angenommen werden, dass hierbei neue Arrangements alltäglicher Lebensführung herausgebildet werden. In Anlehnung an Forschungsarbeiten zur doppelten Entgrenzung von Erwerbsarbeit und Familie (v.a. Jurzcyk et al. 2009), in denen beidseitige strukturelle Veränderungen zusammengebracht, Chancen und Risiken dieser doppelten Entgrenzung identifiziert und individuelle „Grenzmanagementstrategien“ analysiert wurden, wäre eine ähnliche Herangehensweise für die Analyse der doppelten Entgrenzung von Erwerbsarbeit und Konsum denkbar.

Einzelne Hinweise auf veränderte Konsumpraktiken durch entgrenzte Erwerbsarbeit lassen sich in diesem arbeits- und familiensoziologischen Forschungskontext bereits finden. Schließlich sind Familien- und Haushaltsführung eng mit Konsum verbunden. Beispielsweise wird im Zusammenhang mit einer räumlichen Entgrenzung von Erwerbsarbeit und einer daraus teilweise folgenden Multilokalität von Familie auch eine Zunahme an Mobilität, Gütern und beanspruchtem Wohnraum thematisiert (Gräbe / Ott 2003; Schier 2009).

Auch in der sozial-ökologischen (Konsum-)Forschung gibt es Arbeiten, die sich bereits mit den Auswirkungen entgrenzter Erwerbsarbeit auf Konsum beschäftigt haben. Dabei geht es vor allem um die Chancen und Grenzen zur Förderung nachhaltigen Konsums (Hielscher 2000; Hildebrandt 2000; Heimerl 2002). Bislang fehlen jedoch Forschungsarbeiten mit einem systematischen und umfassenden Blick auf das Zusammenspiel von Veränderungen in beiden Bereichen. Es bleibt Aufgabe zukünftiger Forschung, diese Lücke zu schließen und die Entgrenzungen von Erwerbsarbeit und Konsum im Zusammenspiel von alltäglicher Lebensführung zu analysieren.

Dieser Artikel soll einen ersten Beitrag zur Verknüpfung von entgrenzter Erwerbsarbeit und Konsum leisten, indem aufgezeigt wird, dass Unternehmen auf zwei Wegen die Doppelrolle ihrer Mitarbeiter als Konsumenten (Konsumenten-Mitarbeiter) berücksichtigen. In den beiden folgenden Kapiteln werden die Konsumenten-Mitarbeiter einerseits als Adressaten von unternehmerischen Leistungen (Kapitel 3) und andererseits als Akteure der Leistungserbringung (Kapitel 4) näher beleuchtet.

\section{Konsumenten-Mitarbeiter als Adressaten von unternehmerischen Konsumangeboten}

Sämtliche Aktivitäten, die auf den Erwerb, Ge- und Verbrauch, die Entsorgung bzw. Weitergabe von Gütern bzw. auf die Nutzung von Dienstleistungen gerichtet sind und zur individuellen Bedürfnisbefriedigung beitragen sollen, können als Konsum bezeichnet werden (u.a. Scherhorn 1977, 1980; Wiswede 2000; Schrage 2009; Hellmann 2012). Der Ge- und Verbrauch von Ressourcen im weitesten Sinne kann im Arbeits- und Privatleben sehr ähnlich sein. Beispielsweise nutzen Mitarbeiter ebenso Strom und Papier am Arbeitsplatz, wie sie es im Privatleben als Konsumenten auch tun. Während der Papierverbrauch am Arbeitsplatz jedoch Teil der Leistungserbringung im Unternehmen, also der Produktion, ist, gehört der Papierverbrauch im Privatleben zur privaten Bedürfnisbefriedigung, also zum Konsum. Zugleich konsumieren Mitarbeiter aber auch am Arbeitsplatz. Sie naschen Schokolade am Schreibtisch oder machen Online-Shopping am Arbeitsrechner. Hierbei befriedigen sie persönliche Bedürfnisse. Der Ressourcenverbrauch dient nicht dem Produktionsprozess.

Die Unterscheidung zwischen Konsum und Produktion durch eine Bestimmung des Ressourcenverbrauchs als individuelle Bedürfnisbefriedigung oder unternehmerische Leistungserbringung basiert jedoch auf der Trennung eines Kreislaufs. Schließlich ist die durch Konsum aufrechterhaltene und regenerierte Arbeitskraft immer auch notwendige Voraussetzung für den Produktionsprozess (Marx [1867]1962). Dies zeigt sich besonders deutlich bei Kon- 
sumaktivitäten, die der Erwerbsarbeit unmittelbar vor- oder nachgelagert sind bzw. innerhalb von Erwerbsarbeit stattfinden. So lässt sich zumindest diskutieren, auch wenn im Folgenden die genannte Unterscheidung beibehalten wird, ob der konzentrationsfördernde Kaffee kurz vor Arbeitsbeginn oder das schnelle Mittagessen zwischen zwei Arbeitsterminen vorrangig konsum- oder produktionsbezogene Aktivitäten sind.

Unternehmen haben längst erkannt, dass der Konsum von Mitarbeitern eine zentrale Stellschraube zum Erhalt bzw. zur Förderung von psychischer und physischer Arbeitskraft ist. Schon zu Marx' Lebzeiten und in den folgenden Industrialisierungsjahren haben einzelne Unternehmen ihrer Mitarbeiterschaft mit Konsumangeboten, beispielsweise der Organisation von Konsumanstalten oder dem Bau von Mietskasernen geholfen (z.B. Zeppelin Wohlfahrt GmbH 2013; ThyssenKrupp AG 2011).

Heute sind unternehmerische Leistungen, die die Mitarbeiter beim Konsumieren unterstützen, weit verbreitet. Diese Mitarbeiterkonsum-Maßnahmen können in zwei unterschiedliche Bereiche unterschieden werden: Erstens findet Mitarbeiterkonsum im Erwerbsarbeitskontext statt und wird durch entsprechende Maßnahmen dort bewusst berücksichtigt bzw. in diesen integriert. Fast alle Unternehmen adressieren den Konsum am Arbeitsplatz, indem sie eine konkrete Konsuminfrastruktur, zumindest für körperliche Grundbedürfnisse, schaffen. Sie stellen beispielsweise Kantinen, Cafeterien und Teeküchen bereit. Außerdem regeln Unternehmen den Ge- und Verbrauch von unternehmenseigenen Produktionsmitteln und von hergestellten Unternehmensprodukten. Zweitens wird Mitarbeiterkonsum im Privatleben, also außerhalb des konkreten Arbeitsortes und außerhalb der Arbeitszeit, adressiert. An erster Stelle stehen hierbei vielfältige freie und/oder vergünstigte Abgaben von Konsumprodukten in allen Bereichen, die entweder im eigenen Unternehmen produziert (z.B. Haustrunk in Brauereien oder Freifahrten für Bahnmitarbeiter) oder in Kooperation mit Partnerunternehmen angeboten werden und im Privatleben konsumiert werden können. Auch zählt hierzu der Mobilitätskonsum von Mitarbeitern, der zum Beispiel durch Jobtickets im öffentlichen Personennah- und -fernverkehr oder durch Dienstwagen von vielen Unternehmen gefördert wird.

Obwohl der Mitarbeiterkonsum eine fast traditionelle strukturelle Verbindung zwischen Erwerbsarbeit und Privatleben darstellt und seit jeher von Unternehmen berücksichtigt wird, zeigen die Entwicklungen der letzten Jahre eine deutliche Veränderung sowohl im unternehmerischen Umgang mit den Konsumbedürfnissen bzw. -wünschen ihrer Mitarbeiter als auch in den konsumbezogenen Ansprüchen und Vorstellungen der Mitarbeiter. Dies wird vor allem in zwei Entwicklungssträngen deutlich:

Zum einen lässt sich insbesondere bei technischen Geräten beobachten, dass feste Zuordnungen zur Konsum- oder Produktionssphäre zunehmend aufgelöst werden. Voß (1998) hat zwar bereits vor 15 Jahren beschrieben, dass entlang einer sogenannten ,technischen Entgrenzungsdimension“" Arbeitsmittel und Arbeitstechnik, die auch für den Privatgebrauch zugängig gemacht werden, die Entgrenzung von Erwerbsarbeit und Privatleben vorantreiben. Als Beispiele dafür nannte er Dienstwagen und Computer. Auch beschrieb er bereits, dass private Güter als Produktionsmittel von den Mitarbeitern eingesetzt werden, wenn sie beispielsweise ihre privaten Räumlichkeiten oder private Telefone für die Verrichtung von Erwerbsarbeit nutzen (Voß 1998). Dieser technische Entgrenzungsprozess hat sich in den letzten Jahren jedoch vor allem im Bereich von Informations- und Kommunikationstechnik deutlich intensiviert, weil Mobiltelefone, Smart Phones, Laptops oder Tablets nicht nur zentrale Produktionsmittel in vielen Erwerbsarbeitsbereichen geworden sind, sondern gleichermaßen populäre Konsumgüter darstellen. Da Unternehmen mobile Endgeräte für die private Nutzung zur Verfügung stellen oder ihren Mitarbeitern gestatten, ihre eigenen favorisierten Geräte mit zur Arbeit zu bringen bzw. diese zur Verrichtung der Erwerbsarbeitsleistungen zu benutzen, nutzen Mitarbeiter zunehmend dieselben Geräte als Konsum- und Produktions- 
mittel (z.B. AOK Bundesverband et al. 2013). Die Ausbreitung und die Angleichung privaten Technikkonsums an erwerbsarbeitsbezogene Produktionstechnik stellen dabei eine zentrale Voraussetzung und zugleich auch Folge der Intensivierung technischer Entgrenzungsprozesse zwischen Erwerbsarbeit und Privatleben dar. Konsumgüter, die zugleich Produktionsgüter sind, werden damit zu hybriden Verbindungsstücken zwischen Erwerbsarbeit und Privatleben. Sie korrespondieren mit anderen, bspw. zeitlichen oder räumlichen, Entgrenzungsprozessen und ermöglichen bzw. fördern dabei den Einbezug privater Ressourcen und subjektiver Potentiale in den Erwerbsarbeitsprozess.

Zum anderen wird Mitarbeiterkonsum insgesamt in verstärktem Maße sowohl im Erwerbsarbeitskontext als auch im Privatleben von Unternehmen adressiert und fördert dabei die Integration beider Lebensbereiche. Dies wird besonders deutlich in zunehmend populären und als betriebswirtschaftlich relevant anerkannten Unternehmensmaßnahmen zur Unterstützung der Gesunderhaltung und der Vereinbarkeit von „Arbeit und Leben“ (auch „Work-Life-Balance“) (BMFSFJ 2005; Mohe / Dormiok / Kaiser 2010; Badura et al. 2012). In entsprechenden Maßnahmen versuchen Unternehmen, neben anderen Zielen, bestehende Konsumbedürfnisse der Mitarbeiter zu befriedigen, konsumbezogene Aufgaben zu übernehmen oder aber negativen Konsumeffekten entgegenzuwirken. Beispielsweise organisieren Unternehmen Wellnessdienstleistungen (z.B. Massagen oder Yogastunden), bieten eine gesundheitsfördernde Kost an, engagieren Ernährungsexperten oder fördern Suchtberatung und -prävention am Arbeitsplatz (z.B. BMG 2011). Auch unterstützen Unternehmen die Mitgliedschaft in Fitnessstudios und fördern das Fahrradfahren, zum Beispiel durch Kooperationen mit Vereinen oder Krankenkassen (mit-dem-rad-zur-arbeit.de). Es gibt Unternehmen, die, vor allem für ihre Führungskräfte, in Zusammenarbeit mit externen Dienstleistern sogenannte Concierge-Dienste anbieten, die beispielsweise Umzüge organisieren, Einkäufe erledigen oder Reparaturen übernehmen. In diesem Kontext sind auch Konzepte zu sehen, die eine Förderung von sozial-ökologisch verträglichem Konsum von Mitarbeitern zum Ziel haben (Muster 2010; Muster / Schrader 2011; Seebacher / Schultz / Mert 2011).

Work-Life-Balance-Maßnahmen werden zwar bereits im Zusammenhang mit einer Entgrenzung von Erwerbsarbeit diskutiert (Jurczyk 2004; Jürgens 2006; Fleetwood 2007; Dunkel et al. 2010; Kratzer et al. 2011). Bislang wenig beleuchtet wurde dabei aber die Rolle von Konsumgütern und Dienstleistungen. Indem Unternehmen verstärkt Konsumgüter und Dienstleistungen am Arbeitsplatz organisieren, mit Hilfe derer private Konsumwünsche, Bedürfnisse oder auch konsumbezogene Probleme der Mitarbeiter adressiert werden, entsteht mehr „Privatheit" im Erwerbsarbeitsbereich. Zugleich nehmen die Unternehmensbezüge im Privatleben zu, wenn entsprechende Konsumgüter und Dienstleistungen auch außerhalb von Arbeitszeit und -ort genutzt werden können. Unternehmen, die solche Angebote für ihre Mitarbeiter organisieren, nehmen Einfluss auf den Verbrauch, den Erhalt und die (Wieder-)Herstellung von Mitarbeiterressourcen durch Konsum, was mit neuen bzw. veränderten Spielräumen und Verwertungsmöglichkeiten für Mitarbeiter und Unternehmen einhergehen kann.

Unternehmen berücksichtigen die Doppelrolle ihrer Konsumenten-Mitarbeiter jedoch nicht nur, indem sie Konsumangebote für sie bereitstellen. Sie binden die KonsumentenMitarbeiter auch direkt als Akteure der Leistungserbringung in Produktionsprozesse ein. Wie das geschieht, soll im Folgenden vorgestellt werden.

\section{Konsumenten-Mitarbeiter als Akteure der Leistungserbringung}

Personalpolitische Maßnahmen, die zu einer Entgrenzung von Erwerbsarbeit beitragen, fördern, wie in Kapitel 2.1 beschrieben, den Einbezug von subjektiven Potentialen und privaten Ressourcen in die Erwerbsarbeit. Einige dieser Potentiale und Ressourcen stehen in enger Verbindung zur privaten Konsumentenrolle der Mitarbeiter. Als Konsumenten entwickeln 
die Mitarbeiter schließlich bestimmte Persönlichkeitseigenschaften, sammeln Erfahrungen, haben Ideen und Wünsche oder interagieren mit anderen Unternehmen und Konsumenten.

Mit unterschiedlichen Maßnahmen versuchen Unternehmen diese konsumbezogenen Ressourcen ihrer Konsumenten-Mitarbeiter, die das vermutlich teilweise selbst anbieten und einfordern, in die Wertschöpfung zu integrieren. Das passiert auf zwei unterschiedlichen Wegen: Zum einen erhalten die Konsumenten-Mitarbeiter die Möglichkeit, konsumbezogene Potentiale und Ressourcen direkt in Arbeitsprozesse einzubinden, zum Beispiel ihre privaten Produkterfahrungen oder konsumspezifisches Wissen. Zum anderen werden die Mitarbeiter dabei unterstützt, Erwerbsarbeitsleistungen im privaten Konsumbereich zu erbringen, zum Beispiel als Mundwerber oder Beta-Tester. Die Mitarbeiter sind hierbei als Konsumenten produktiv.

Mitarbeiter, die als produktive Konsumenten in Unternehmen tätig sind, weisen zu Kunden, die ebenfalls als produktive Konsumenten für Unternehmen tätig sind (siehe Kapitel 2.2), große Ähnlichkeiten auf. Hierbei zeigt sich somit ein Zusammenhang zwischen der Entgrenzung von Erwerbsarbeit und der Entgrenzung von Konsum. Während in beiden Bereichen Konsumenten produktiv sind und diese Produktivkraft in Unternehmensprozesse einfließt, besteht der Unterschied vor allem in der Art der Geschäftsbeziehung zwischen Personen und Unternehmen - als Mitarbeiter oder Kunde.

Diese „konsumspezifische Subjektivierung“ von Erwerbsarbeit wurde aus arbeits- und industriesoziologischer Sicht bislang noch nicht beleuchtet. Sie stellt eine neue, spezifische Facette subjektivierter Erwerbsarbeit dar. Verschiedene betriebswirtschaftliche Ansätze zeigen jedoch deutlich auf, dass die Leistungspotentiale von Konsumenten-Mitarbeitern bereits erkannt wurden. Im Folgenden sollen drei unterschiedliche Bereiche vorgestellt werden, in denen auf die konsumbezogene Produktivkraft von Mitarbeitern als Markenbotschafter (4.1), als Innovatoren (4.2) und als Umweltengagierte (4.3) abgezielt wird.

\subsection{Mitarbeiter als Markenbotschafter}

Für die glaubwürdige Kommunikation einer Produkt- oder Unternehmensmarke ist es von zentraler Bedeutung, wie die Mitarbeiter agieren und kommunizieren (Harris / de Chernatony 2001; Müller-Neuhoff / Giehl 2004; Morhart / Herzog / Tomczak 2009; Henkel et al. 2007, Henkel / Tomczak / Wentzel 2011). Die Mitarbeiter machen die Marke konkret und füllen sie mit Leben (Henkel / Tomczak / Wentzel 2011). Daher kommt es darauf an, dass sich die Mitarbeiter markenkonform, also im Sinne des Markenversprechens, verhalten (Henkel et al. 2007). Mit Hilfe von „Internal Branding“- bzw. „Behavioural Branding“Maßnahmen wird versucht, das Mitarbeiterverhalten entsprechend zu beeinflussen.

Henkel et al. (2007) sowie Henkel, Tomczak und Wentzel (2011) weisen darauf hin, dass es vor allem auf informelle Maßnahmen und Empowerment ankommt, um die Mitarbeiter zu markenkonformen Verhalten zu bewegen. Beides sind Maßnahmen, die eine Entgrenzung von Erwerbsarbeit fördern. Bei ersterer geht es um den persönlichen Austausch zwischen Vorgesetzten und Mitarbeitern. Beim Empowerment steht im Vordergrund, dass die Mitarbeiter eigenständiger und selbstverantwortlicher handeln können (und sollen), mehr Befugnisse erhalten und Einfluss ausüben können (Spreitzer 1995). Auch Morhart, Herzog und Tomczak (2009) betonen, dass Freiraum zur Ausgestaltung der Arbeitsrolle und individualisierte Unterstützung durch die Vorgesetzen wichtig sei. Die daraus folgende Stärkung von Verbundenheit und Autonomie ,would ultimately spill over into the commitment, authenticity, and proactivity that characterize a real brand champion" (Morhart / Herzog / Tomczak 2009: 138). 
Markenkonformes Verhalten der Mitarbeiter wird nicht nur im Arbeitskontext als bedeutsam angesehen:

„, Mitarbeiter sind Botschafter der Marke 24 Stunden am Tag, sieben Tage in der Woche. Denn natürlich werden die Mitarbeiter im privaten Umfeld, in der Familie und im Freundeskreis angesprochen auf ihr Unternehmen als Arbeitgeber, hinsichtlich der Qualität von Produkten und Dienstleistungen [...] " (Kernstock 2008: 23).

Markenkonformes Verhalten der Mitarbeiter ist daher in deren Arbeits- und Privatleben von Bedeutung für Unternehmen. Ob und inwiefern es Mitarbeitern gelingt, sich in Arbeits- und Privatleben auch tatsächlich markenkonform zu verhalten, hängt jedoch entscheidend von ihrem Lebensstil bzw. von ihrem eng mit dem Lebensstil verbundenen Konsumverhalten ab (Lüdke 2000). Ein Mitarbeiter im Verkaufsgespräch für eine Harley Davidson ist sicherlich überzeugender, wenn er von seiner letzten Reise mit der eigenen Harley erzählt, als wenn er privat einen anderen Motorradtyp bevorzugt oder sogar in seiner Freizeit lieber Fahrrad fährt. Ein Mitarbeiter im Bio-Laden kann wahrscheinlich dann am besten beraten, wenn er auch privat Bio-Produkte konsumiert und persönlich von deren Nutzen für Umwelt und Gesellschaft überzeugt ist.

Der Logik von „Behavioural Branding“ folgend liegt es deshalb nahe, dass auch das Konsumverhalten der Mitarbeiter zur Marke passen bzw. entsprechend angepasst werden sollte. Unternehmerische Konsumangebote für Mitarbeiter, die in Kapitel 3 bereits dargestellt wurden, können deshalb auch in diesem Kontext einen zentralen Beitrag leisten und zum Aufbau von Mitarbeiterressourcen, wie markenrelevanten Konsumerfahrungen und -kompetenzen, beitragen. Die in vielen Unternehmen übliche freie oder vergünstigte Abgabe von in Unternehmen selbst hergestellten Produkten erscheint deshalb vor dem Hintergrund von „Behavioural Branding “äußerst fruchtbar, weil dadurch die Identifikation und Erfahrung der Mitarbeiter mit den eigenen Unternehmensprodukten gesteigert werden können. Aber auch andere von Unternehmen organisierte Konsumangebote oder Dienstleistungen für Mitarbeiter sind hierbei hilfreich. So kann beispielsweise vermutet werden, dass Unternehmen, deren Marke mit Fitness und Dynamik assoziiert ist, im Rahmen von „Behavioural Branding“ ihre Mitarbeiter dabei unterstützen, durch entsprechende Sport- und Ernährungsangebote ebenfalls körperlich fit und dynamisch zu sein. Auch ist denkbar, dass Unternehmen, deren Markenkern mit Umweltschutz assoziiert ist, ihre Mitarbeiter dabei unterstützen, umweltfreundlich zu konsumieren (z.B. durch Bio-Produkte in der Kantine oder Fahrradaktionstage).

Es wird deutlich, dass im Konsum der Mitarbeiter ein relevantes Leistungspotential für Unternehmen steckt. Ein markenkonformes (Konsum)-Verhalten der Mitarbeiter erfüllt zusammengefasst demnach zwei zentrale Funktionen: Erstens hilft es dabei, dass Mitarbeiter bessere, nämlich authentischere und damit glaubwürdigere Markenbotschafter im Arbeitskontext sind. Zweitens fördert ein markenkonformes (Konsum-)Verhalten, dass die Mitarbeiter auch als Privatpersonen positiv und glaubwürdig über die Marke und das Unternehmen kommunizieren. In ihrer Doppelrolle als Konsumenten-Mitarbeiter machen sie wirksame Mundwerbung in ihren (digitalen) sozialen Netzwerken und tragen somit zu einem positiven Image und zur Bekanntheit des Unternehmens und seiner Produkte bei.

\subsection{Mitarbeiter als Innovatoren}

Innovationsprozesse, an denen nicht nur die Mitarbeiter einer Forschungsabteilung arbeiten, sondern auch andere Personen, beispielsweise Kunden oder Wissenschaftler, beteiligt sind, werden „Open Innovation“ genannt (u.a. von Hippel 1988; Chesbrough 2003; Reichwald et al. 2007; Enkel / Gassmann / Chesbrough 2009; Koch / Bullinger / Möslein 2009; Neyer / Bullinger / Möslein 2009). Kunden haben ein besonderes Bedürfnis- und Problemwissen 
durch ihre Nutzungserfahrungen mit den Produkten und oftmals zugleich auch Lösungsideen, wie sich ihre Bedürfnisse am besten befriedigen ließen (Reichwald et al. 2007).

Auch Mitarbeiter anderer Abteilungen können in den Innovationsprozess einbezogen werden (Koch / Bullinger / Möslein 2009; Neyer / Bullinger / Möslein 2009). Diese Mitarbeiter sind zwar ,not responsible for innovative activity by their job description, but nonetheless [they are] interested in and ha[ve] the potential to produce innovative ideas and contribute to the innovation process by suggesting, supporting, or refining innovative concepts" (Neyer / Bullinger / Möslein 2009: 411). Diese ,peripheren Innovatoren“ können den Innovationsprozess mit dem Wissen von anderen Stufen des Wertschöpfungsprozesses (z.B. Produktion oder Marketing) anreichern. Das spezifische Bedürfnis- und Problemwissen von Konsumenten und das Wissen aus verschiedenen Stufen des Wertschöpfungsprozesses bringen solche Mitarbeiter zusammen, die auch Konsumenten mit relevanten Produkterfahrungen sind.

Schrader und Belz (2011) weisen darauf hin, dass ,,insbesondere für Konsumgüterhersteller [...] die Einbindung von Mitarbeiter/innen als Konsument/innen bzw. als Nutzer/innen neue Möglichkeiten schaffen“ kann, da sich hier „das Kreativitätspotential der klassischen Nutzerintegration und die Sicherstellung der Relevanz für Unternehmen [ergänzen]" (Schrader / Belz 2011: 344). Schweisfurth (2013) arbeitet empirisch heraus, welche für Innovationsprozesse relevanten Eigenschaften Mitarbeiter als Konsumenten mitbringen. Dabei fokussiert er auf Mitarbeiter, die „trendführende Konsumenten“ bzw. Lead User sind. Er zeigt, dass nicht nur das konsum- und produktionsspezifische Wissen eine wichtige Rolle spielen. Die Mitarbeiter, die zugleich Lead User sind, genießen durch ihre Praxiserfahrungen auch eine hohe Vertrauens- und Glaubwürdigkeit im Unternehmen. Sie haben Zugang zu und Verständnis für beide Akteursgruppen und besitzen die Fähigkeit, die unterschiedlichen Perspektiven der zwei Bereiche zusammenbringen.

Der Konsum der Mitarbeiter wird hierbei zu einer zentralen Produktivitätsquelle: So bringen die Mitarbeiter Konsumerfahrungen und konsumspezifische Bedürfnisse in Unternehmensprozesse ein. Dabei greifen sie nicht nur auf ihre eigenen Konsumerfahrungen zurück. Ihr Austausch mit anderen Konsumenten im Privatleben (z.B. User Communities oder Fanklubs, Marschall 2010) ermöglicht es ihnen, auch deren spezifische Erfahrungen und Bedürfnisse zu sammeln und in die unternehmerischen Innovationsprozesse zu integrieren. Ihre Konsumerfahrungen im Privatleben fördern zudem ihre Glaubwürdigkeit und Kompetenz als innovierender Mitarbeiter im Unternehmen.

Auch können Mitarbeiter als Konsumenten erste Ideen und Prototypen im realen Konsumumfeld weiterentwickeln bzw. selbst testen und ihre Erfahrungen ins Unternehmen zurückspiegeln. Solche Praktiken sind beispielsweise aus der Autoindustrie oder bei der Softwareentwicklung bekannt, wo Mitarbeiter als Konsumenten frühzeitig in Testphasen, quasi als Beta-Tester, eingebunden werden. Zudem ist denkbar, dass Mitarbeiter als Konsumenten auch bei der Diffusion von Produktinnovationen helfen, indem sie gezielt Informationen über und Erfahrungen mit den neuen Produkten in ihren privaten sozialen Netzwerken verbreiten (Schmidt 2013).

Die Verknüpfung von „entgrenztem Konsum“ und „entgrenzter Erwerbsarbeit“ zeigt sich im Kontext von Open Innovation somit besonders stark. Kunden werden, wie bereits dargestellt, schließlich schon längst als „Lead User“ in Innovationsprozesse eingebunden.

\subsection{Mitarbeiter als Umweltengagierte}

Angenommen werden kann, dass Unternehmen, die ihre Produkte und Prozesse sozial und ökologisch verträglich(er) gestalten wollen, darauf angewiesen sind, dass sie Mitarbeiter haben, die ebensolche Werte vertreten und entsprechend handlungskompetent sind. Schrader 
und Harrach (2013) argumentieren deshalb, dass Mitarbeiter, die sich im Privatleben bereits für Umwelt- bzw. Nachhaltigkeitsthemen interessieren und „bewusste“ Konsumenten sind, nicht erst überzeugt und motiviert werden müssen. Im Gegenteil: anzunehmen ist, dass solche Konsumenten ihre nachhaltigkeitsbezogenen Einstellungen und Verhaltensweisen auch gern am Arbeitsplatz verwirklichen möchten und dafür eine besondere Motivation und Handlungsbereitschaft mitbringen (Muster / Schrader 2011; Schrader / Harrach 2013; Harrach et al. 2014).

Umweltorientierte Konsumenten sind schließlich meist sehr interessierte und achtsame Konsumenten, sie kennen sich gut mit Produkten aus und sie sprechen auch gern mit anderen über ihr Wissen und ihre Meinungen (Shrum / McCarty / Lowrey 1995). Altruistische Motive sind bei ihnen stärker ausgeprägt als bei anderen Konsumentengruppen und sie glauben, mit ihren Handlungen etwas bewegen zu können (Akehurst / Afonso / Goncalves 2012).

Vorgeschlagen wird deshalb, dass diese umwelt- und nachhaltigkeitsorientierten Mitarbeiter als "sustainable intrapreneurs“ besonders gefördert werden sollen (Schrader / Harrach 2013). Indem sie größere Handlungsspielräume und Eigenverantwortung im Arbeitsprozess erhalten, sollen sie dazu in die Lage versetzt werden, die Umsetzung von Umweltschutz und Nachhaltigkeit im Unternehmen voranzutreiben.

Ein ähnlicher Ansatz wird im Projekt „enEEbler“ verfolgt, in dem es um ein Mitarbeiterengagement für Erneuerbare Energien geht (Alanus Hochschule 2013). Hier ist der Ausgangspunkt die Annahme, dass Mitarbeiter, die sich bereits privat mit Erneuerbaren Energien beschäftigen, eine alternative Energieversorgung selbst nutzen oder in einer privaten Initiative für Erneuerbare Energien aktiv sind, dieses (zu Teilen konsumbezogene) Engagement auch in Unternehmen tragen und dort die Umstellung auf Erneuerbare Energien vorantreiben können. Ziel des Forschungsprojektes ist es deshalb, herauszufinden, unter welchen Umständen Mitarbeiter ihr privates Engagement auch auf den Arbeitsplatz übertragen und mit welchen Maßnahmen diese Übertragung gefördert werden kann (Herbes 2013).

Die Potentiale von umweltengagierten Konsumenten sollen nach Schrader und Harrach (2013) sowie Herbes (2013) demnach auf zwei Wegen verwertet werden: Einerseits geht es darum, die Motivationen für verändertes Konsumverhalten zu nutzen und die Konsumerfahrungen der Mitarbeiter ins Unternehmen zu integrieren. Wenn es tatsächlich gelingt, die Handlungsbereitschaft, die Erfahrungen und das Wissen der Konsumenten-Mitarbeiter auch auf den Arbeitsplatz zu übertragen, ist eine wichtige Voraussetzung geschaffen, damit auch entsprechende Unternehmensaktivitäten vorangebracht werden können. Andererseits können Mitarbeiter als umweltengagierte Konsumenten dabei helfen, Kontakte und Kooperationen nach außen herzustellen bzw. Projekte mit sozial-ökologischen Akteuren ihres privaten Umfeldes (z.B. Initiativen oder Vereinen) zu realisieren.

Zusammenfassend lässt sich für die drei vorgestellten Bereiche Folgendes feststellen: Den Mitarbeitern werden durch verschiedene Maßnahmen mehr Freiraum und Verantwortung im Arbeitsprozess zugeschrieben, was die Integration subjektiver Potentiale und privater Ressourcen möglich und zugleich auch nötig macht. Diese Potentiale und Ressourcen haben bei Mitarbeitern als Markenbotschafter, Innovatoren oder Umweltengagierte einen spezifischen Konsumbezug. Die Mitarbeiter werden dabei in ihrer Doppelrolle als Konsumenten-Mitarbeiter adressiert und erbringen in dieser Doppelrolle erwerbsarbeitsbezogene Leistungen entweder am Arbeitsplatz oder aber im privaten Konsumbereich.

\section{Folgen für Konsumenten-Mitarbeiter}

Wie in Kapitel 2 skizziert, geht die Entgrenzung und Subjektivierung von Erwerbsarbeit, aber auch die Entgrenzung von Konsum mit Chancen und Risiken für Personen einher. Da 
die unternehmerische Berücksichtigung des Mitarbeiterkonsums und der darin liegenden Potentiale eine spezifische Facette subjektivierter Erwerbsarbeit darstellt, können teilweise ähnliche Chancen und Risiken für Konsumenten-Mitarbeiter vermutet werden, wie sie für Erwerbstätige im Kontext subjektivierter Erwerbsarbeit allgemein formuliert wurden (siehe dazu Voß 1998; Voß / Pongratz 1998; Kleemann / Matuschek / Voß 1999; Kratzer 2003; Pongratz / Voß 2003; Jürgens 2006; Moosbrugger 2012 und Kapitel 2.1). Es können aber auch neue bzw. veränderte Chancen und Risiken angenommen werden. Vor diesem Hintergrund sollen im Folgenden die Chancen und Risiken für Konsumenten-Mitarbeiter, die als Adressaten von Konsumangeboten (Kapitel 3) und als Akteure der Leistungserbringung (Kapitel 4) von Unternehmen berücksichtigt werden, illustriert werden.

\subsection{Chancen für Konsumenten-Mitarbeiter}

Konsumangebote stellen zusätzliche bzw. erweiterte Handlungsoptionen für Mitarbeiter als Konsumenten dar. Indem Unternehmen die Gestaltung der Vor-Nutzungsphase übernehmen, senken sie Anbahnungs-, Informationsbeschaffungs- und Vereinbarungskosten für Mitarbeiter. Die Bereitstellung von Konsumangeboten zur Nutzung am Arbeitsplatz oder im näheren Umfeld des Arbeitsplatzes senkt Zeit- und Wegekosten. Die Angebote sind zudem auch finanziell attraktiv, da sie oftmals kostenlos oder rabattiert verfügbar sind. Da auch Kollegen und Vorgesetzte die Angebote erhalten bzw. in Anspruch nehmen, entstehen gemeinsame Erlebnisse und verbindende Gesprächsthemen in der Mitarbeiterschaft.

Die durch die Konsumangebote verstärkte Integration von Erwerbsarbeit und Privatleben kann von Mitarbeitern durchaus als positiv und hilfreich empfunden werden. Da unternehmerische Gesundheits- und Vereinbarkeitsmaßnahmen auch als Antwort auf erhöhte Anforderungen durch entgrenzte Arbeitsbedingungen diskutiert werden (Jurzcyk 2005; Jürgens 2006; Dunkel et al. 2010; Kratzer et al. 2011), kann angenommen werden, dass auch in diesem Kontext organisierte Konsumangebote dazu beitragen können, die Gesundheit und Vereinbarkeit zwischen Erwerbsarbeit und Privatleben zu fördern.

Wenn technische Produktionsmittel für den privaten Konsum verfügbar gemacht werden und damit den Mitarbeiterbedürfnissen entsprechen, können schließlich das Wohlbefinden und die Zufriedenheit der Mitarbeiter gesteigert werden. Einigen Mitarbeitern ist es erst durch die Bereitstellung der Geräte für private Zwecke möglich, auch als Konsumenten von deren Nutzen zu profitieren.

Die Möglichkeit, individuelle Konsumerfahrungen oder Konsumwissen in die Erwerbsarbeit einzubringen, kann, wie auch beim Einbezug anderer persönlicher Potentiale, den Subjektivierungsansprüchen von Mitarbeitern entsprechen und ihre Erwerbsarbeit angenehmer machen. Gerade im Konsum manifestieren sich schließlich der Lebensstil und das Selbstbild von Personen.

Die unternehmerische Wertschätzung privater Konsumerfahrungen und Fähigkeiten der Mitarbeiter kann außerdem, wie auch bei Kunden, die zum Beispiel im Rahmen von Crowdsourcing in Unternehmensprozessen mitarbeiten, das Selbstwertgefühl steigern und Selbstwirksamkeitserfahrungen fördern. Wie Schweisfurth (2013) zeigt, können KonsumentenMitarbeiter schließlich auch ihren Status im Unternehmen, beispielsweise indem sie an Glaubwürdigkeit und wahrgenommener Kompetenz gewinnen, verbessern, was positive Effekte auf Arbeitszufriedenheit und Wohlbefinden haben kann. Mehr noch, es konnte bereits gezeigt werden, dass Mitarbeiter, gerade erst weil sie sich auch in ihrer Konsumentenrolle im Erwerbsarbeitsalltag wahrgenommen und anerkannt fühlen, zufrieden(er) und motiviert(er) fühlen (Schrader / Harrach / Stanszus 2013; Harrach et al. 2014). Gleichwohl profitieren nicht alle Mitarbeiter von diesen Chancen. 


\subsection{Risiken für Konsumenten-Mitarbeiter}

Die Entgrenzung von Erwerbsarbeit und Privatleben durch Konsumangebote oder die Integration von Konsumkompetenzen ist vor allem dann problematisch, wenn es Mitarbeitern nicht gelingt, diese Entgrenzungsprozesse selbst zu gestalten bzw. zu steuern. Ein erfolgreiches „Grenzmanagement“" zwischen Erwerbsarbeit und Privatleben setzt schließlich verschiedene Kompetenzen und Persönlichkeitseigenschaften, aber auch entsprechende Rahmenbedingungen in Erwerbsarbeit und Privatleben voraus (Jürgens 2006).

Mitarbeiter müssen sehr genau abwägen, welche Signale sie mit einem bestimmten Nutzungs- oder Ablehnungsverhalten von Konsumangeboten an ihre Kollegen und Vorgesetzten senden. Angenommen werden kann, dass viele solcher Maßnahmen nicht von den Mitarbeitern aktiv eingefordert, sondern vom Unternehmen initiiert werden. Während Unternehmen sehr genau bemessen, mit welchen Konsumangeboten sie welche Ziele verfolgen, ist fraglich, inwieweit Mitarbeiter in der Lage sein können, ihren individuellen Nutzen durch die Inanspruchnahme von Konsumangeboten oder die Integration privater Konsumerfahrungen den für sie oftmals weniger offensichtlich entstehenden Kosten gegenüberzustellen und bewusst abzuwägen.

Attraktive Konsumangebote am Arbeitsplatz und im Privatleben können Mitarbeiter dazu bringen, länger, lieber und besser zu arbeiten. Das kann natürlich ganz und gar den Mitarbeiterinteressen entsprechen. Eine dabei entstehende Kultur der Leistungsbereitschaft und -steigerung entspricht jedoch vor allem den Unternehmensinteressen. Auch ein Arbeitsplatz mit den besten Konsum-Rahmenbedingungen bleibt ein Arbeitsplatz für die Mitarbeiter.

Produktionsmittel, die zugleich auch zum privaten Konsum genutzt werden können, erleichtern nicht nur privates Vergnügen, sondern vor allem auch erwerbsarbeitsbezogene Leistungen im Privatleben (z.B. Voß 1998). So fördern privat nutzbare Smart Phones und Laptops die Erreichbarkeit und Verfügbarkeit auch nach Dienstschluss und damit psychische Belastungen (AOK Bundesverband et al. 2013).

Vermutet werden kann, dass gerade unternehmerische Konsumangebote eine besonders kritische Rolle spielen, wenn diskutiert wird, dass Work-Life-Balance-Maßnahmen Belastungen hervorbringende Entgrenzungsprozesse teilweise nicht kompensieren, sondern gerade erst ermöglichen. Einerseits liegt nahe, dass beispielsweise Leistungsdruck nicht durch wöchentliche Massageangebote ausgeglichen werden kann. Wie Kratzer et al. (2011) gezeigt haben, sollten Work-Life-Balance-Angebote, damit sie eine entlastende Wirkung haben, mit Anpassungen in den betrieblichen Leistungsanforderungen bzw. mit arbeitsorganisatorischen Veränderungen einhergehen. Andererseits bergen gerade attraktive Konsumangebote die Gefahr, von notwendigen, strukturellen Veränderungen abzulenken oder Mitarbeiter, die unzufrieden sind, zu besänftigen. Im Unterschied zu arbeitsorganisatorischen Veränderungen können Konsumangebote besonders leicht organisiert und zur Verfügung gestellt werden. Zudem können Konsumangebote und Dienstleistungen gerade dazu führen, dass dadurch frei werdende Ressourcen nicht in andere private Aktivitäten verlagert, sondern in den Erwerbsarbeitsbereich integriert werden. So ist beispielsweise denkbar, dass wegfallende Wegezeiten durch die Inanspruchnahme von Dienstleistungen am Arbeitsort statt in Freizeit in Arbeitszeit verwandelt werden.

Maßnahmen zur Integration privater Konsumerfahrungen sind vor allem für jene Mitarbeiter riskant, die zu einer verstärkten Verzahnung von Erwerbsarbeit und Privatleben nicht bereit sind und/oder nicht über unternehmensrelevante Konsumerfahrungen und Kompetenzen verfügen. 
Im Unterschied zu Kunden, die Unternehmen beim Crowdsourcing freiwillig bei Produktverbesserungen oder Innovationen helfen, haben Mitarbeiter als produktive Konsumenten nur scheinbar eine Wahl. Naheliegend ist schließlich, dass Mitarbeiter schnell unter Druck geraten können, ihre Konsumerfahrungen in Erwerbsarbeitsprozesse einzubringen, wenn sie bemerken, dass sie andernfalls beispielsweise weniger innovativ oder weniger erfolgreich in der Leistungserbringung sind und folglich Karriereeinschränkungen befürchten müssen. Andere Mitarbeiter, die nicht oder nur wenig über relevante Konsumerfahrungen verfügen, können dieses Leistungsdefizit kaum kompensieren. Dies ist beispielsweise denkbar, wenn Mitarbeiter einen Konsumstil pflegen, der nur schlecht zum Unternehmen passt. Sie müssen damit rechnen, dass ihnen bestimmte positive Erwerbsarbeitserlebnisse verwehrt bleiben (z.B. Reputationszuwachs) bzw. sogar negative Konsequenzen (z.B. geringere Prämien) hinnehmen.

Zudem ist naheliegend, dass Unternehmen, je nach Kerngeschäft, auf unterschiedliche Konsumkompetenzen und Erfahrungen abzielen. Wechselnde Arbeitsverhältnisse machen es für Mitarbeiter jedoch schwer, wenn nicht unmöglich, ihre Konsumkompetenzen an jeweils neue Arbeitsanforderungen anzupassen. Gleichwohl ist denkbar, dass sich manche Mitarbeiter gedrängt fühlen, relevante Konsumerfahrungen im Privatleben zu sammeln und deshalb ihren Konsumstil anpassen, damit sie bessere Arbeitsleistungen erbringen können.

\section{Fazit}

Die Doppelrolle vieler Personen als Erwerbsarbeitskraft und Konsument findet in den Analysen zu „entgrenzter Erwerbsarbeit“ und „entgrenztem Konsum“ bislang kaum Beachtung. Unternehmen berücksichtigen ihre Mitarbeiter jedoch längst als Konsumenten.

Im Kontext entgrenzter Erwerbsarbeit hat der Konsum von Mitarbeitern zwei zentrale Funktionen, die in diesem Artikel vorgestellt wurden. Entsprechende Unternehmensmaßnahmen haben amivalente Folgen für Mitarbeiter, wie die Diskussion der Chancen und Risiken gezeigt hat.

Der These von Voß und Rieder (2005), nach der die Entgrenzung von Erwerbsarbeit und die Entgrenzung von Konsum einer komplementären Logik entspringen, lässt sich mit Blick auf Konsumenten-Mitarbeiter, die wie Kunden, durch ihre Konsumerfahrungen Wertschöpfungsprozesse anreichern, Nachdruck verleihen. Gerade diese neue, spezifische Form entgrenzter Erwerbsarbeit gilt es in zukünftigen Forschungsarbeiten genauer zu untersuchen. Welche Erfahrungen machen Mitarbeiter mit Maßnahmen zur Integration von Konsumkompetenzen, die nicht in der Lage sind oder sein wollen, diese Erfahrungen und Kompetenzen in die Erwerbsarbeit zu tragen? Wie beeinflussen Maßnahmen zur Integration von Konsumkompetenzen oder unternehmerische Konsumangebote das Konsumverhalten? Diese und andere Fragen werden zu klären sein.

\section{Literatur}

Akehurst, Gary / Carolina Afonso / Helena M. Goncalves (2012): Re-examining green purchase behaviour and the green consumer profile: new evidences, in: Management Decision 50, S. 972-988.

Alanus Hochschule für Kunst und Gesellschaft (2013): Projektwebsite Eneebler, abrufbar unter: http:// www.eneebler.de/, letztes Abrufdatum: 8.8.2014.

AOK Bundesverband / BKK Bundesverband / Deutsche Gesetzliche Unfallversicherung / Verband der Ersatzkassen e.V. (2013): Auswirkungen von ständiger Erreichbarkeit und Präventionsmöglichkeiten, Teil 1. Initiative Gesundheit und Arbeit, iga-Report 23, abrufbar unter: http://www.iga-info.de/filead$\mathrm{min} /$ Veroeffentlichungen/iga-Reporte_Projektberichte/iga-Report_23_Staendige_Erreichbar-

keit_Teil 1.pdf, letztes Abrufdatum: 8.8.2014. 
Badura, Bernhard / Antje Ducki / Helmut Schröder / Joachim Klose / Markus Meyer (Hrsg.) (2012): Fehlzeiten-Report 2012, Schwerpunktthema: Gesundheit in der flexiblen Arbeitswelt: Chancen nutzen, Risiken minimieren, Berlin.

Blättel-Mink, Birgit / Kai-Uwe Hellmann (2010) (Hrsg.): Prosumer Revisited. Zur Aktualität einer Debatte, Wiesbaden.

Bundesministerium für Familie, Senioren, Frauen und Jugend (BMFSFJ) (2005): Work-Life-Balance. Motor für wirtschaftliches Wachstum und gesellschaftliche Stabilität. Analyse der volkswirtschaftlichen Effekte: Zusammenfassung der Ergebnisse, Berlin.

Bundesministerium für Gesundheit (BMG) (2011): Betriebliche Gesundheitsförderung - Best Practice Beispiele, abrufbar unter: http://www.bmg.bund.de/praevention/betriebliche-gesundheitsfoerderung/ best-practice-beispiele-im-ueberblick.html, letztes Abrufdatum: 8.8.2014.

Chesbrough, Henri W. (2003): Open Innovation. The new imperative for creating and profiting from technology, Boston / MA.

Dunkel, Wolfgang / Wolfgang Menz / Sarah Nies / Dieter Sauer (2010): Einschätzungen zum Forschungsstand Arbeit und Leben (,Work-Life-Balance“), in: IG Metall Vorstand, Arbeitskreis Arbeitspolitik und Arbeitsforschung (Hrsg.), Beiträge zur Arbeitspolitik und Arbeitsforschung, Frankfurt / Main, S. 44-50.

Enkel, Ellen / Oliver Gassmann / Henry Chesbrough (2009): Open R\&D and open innovation: exploring the phenomenon, in: R\&D Management 39, S. 311-316.

Fleetwood, Steve (2007): Why work-life balance now?, in: The International Journal of Human Resource Management 18, S. 387-400.

Gottschall, Katrin / Gerd G. Voß (2005) (Hrsg.): Entgrenzung von Arbeit und Leben. Zum Wandel der Beziehung von Erwerbstätigkeit und Privatsphäre im Alltag, München.

Gräbe, Silvia / Erich Ott (2003): „,...man muss alles doppelt haben.“ Wochenpendler mit Zweithaushalt am Arbeitsort, Münster.

Grün, Oscar / Jean-Claude Brunner (2002): Der Kunde als Dienstleister - Von der Selbstbedienung zur Co-Produktion, Wiesbaden.

Grün, Oscar / Jean-Claude Brunner (2003): Wenn der Kunde mit anpackt - Wertschöpfung durch CoProduktion, in: Zeitschrift Führung + Organisation 72, S. 87-93.

Hanekop, Heidemarie / Volker Wittke (2008): Die neue Rolle der Anwender in internetbasierten Innovationsprozessen, in: Arbeits- und Industriesoziologische Studien 1, S. 7-28.

Hanekop, Heidemarie / Volker Wittke (2012): Nutzergenerierte Beratungsplattformen: Neue Formen der Ko-Produktion im Web 2.0, in: Frank Kleemann / Christian Eismann / Tabea Beyreuther / Sabine Hornung / Katrin Duske / G. Günter Voß (Hrsg.), Unternehmen im Web 2.0. Zur strategischen Integration von Konsumentenleistungen durch Social Media, Frankfurt Main - New York / NY, S. $207-240$.

Harrach, Christoph / Ulf Schrader / Laura Stanszus / Viola Muster (2014): Nachhaltigkeits-Empowerment am Arbeitsplatz lohnt sich. Studie zu nachhaltigkeitsorientierten Mitarbeitern, in: Ökologisches Wirtschaften 1, S. 12-13.

Harris, Fiona / Leslie de Chernatony (2001): Corporate branding and corporate brand performance, in: European Journal of Marketing 35, S. 441-456.

Heimerl, Angelika (2002): Strukturwandel der Arbeit und nachhaltiger Konsum, in: Karl W. Brand / Robert Gugutzer / dies. / Alexander Kupfahl (Hrsg.), Sozialwissenschaftliche Analysen zu Veränderungsmöglichkeiten Nachhaltiger Konsummuster, Unesco Verbindungsstelle für Umwelterziehung im Umweltbundesamt, Dessau-Roßlau, S. 75-99.

Hellmann, Kai-Uwe (2012): Konsum. Eine kurze Bestandsaufnahme aus Sicht der Forschung, in: Praxis Politik 6, S. 4-9. 
Henkel, Sven / Torsten Tomczak / Marc Heitmann / Andreas Herrmann (2007): Managing brand consistent employee behaviour: relevance and managerial control of behavioural branding, in: Journal of Product \& Brand Management 16, S. 310-320.

Henkel, Sven / Torsten Tomczak / Daniel Wentzel (2011): Bringing the brand to life: Structural conditions of brand-consistent employee behavior, in: Thexis 1, S. 13-16.

Herbes, Carsten (2013): Forschungsprojekt "enEEbler": Mitarbeiter-Engagement für Erneuerbare Energien in Unternehmen. Kurzvorstellung bei der ABSI, 26.01. in Hof, abrufbar unter: http://www.solarinitiativen.de/wp-content/uploads/Prof.-Carsten-Herbes-Forschungsprojekt-enEEbler-Mitarbeiter-Engagement.pdf, letztes Abrufdatum: 8.8.2014.

Hielscher, Volker (2000): Erwerbsarbeit im Wandel: Impulse für eine nachhaltige Lebensführung? Flexibilisierung als Barriere, in: Ökologisches Wirtschaften 1, S. 21-23.

Hildebrand, Eckard (2000): Flexible Arbeit und nachhaltige Lebensführung, in: Eckard Hildebrandt (Hrsg.) (in Zusammenarbeit mit Gudrun Linne), Reflexive Lebensführung. Zu den sozialökologischen Folgen flexibler Arbeit, Berlin.

Hippel, Eric v. (1988): The sources of innovation, Oxford.

Jurczyk, Karin (2004): Work-Life-Balance und geschlechtergerechte Arbeitsteilung. Alte Fragen neu gestellt, in: Gudrun Linne (Hrsg.), Flexible Arbeitszeit und soziale Sicherheit, Arbeitspapier 97, Düsseldorf, S. 43-56

Jurczyk, Karin / Maria S. Rerrich (Hrsg.) (1993): Die Arbeit des Alltags. Beiträge zu einer Soziologie der alltäglichen Lebensführung, Freiburg.

Jurczyk, Karin / Michaela Schier / Peggy Szymenderski / Andreas Lange / G. Günter Voß (2009): Entgrenzte Arbeit - entgrenzte Familie. Grenzmanagement im Alltag als neue Herausforderung, Berlin.

Jürgens, Kerstin (2006): Arbeits- und Lebenskraft. Reproduktion als eigensinnige Grenzziehung, Wiesbaden.

Kernstock, Joachim (2008): Behavioral Branding als Führungsansatz. Mit Behavioral Branding das Unternehmen stärken, in: Torsten Tomczak / Franz-Rudolf Esch / ders. / Andreas Herrmann (Hrsg.), Behavioral Branding. Wie Mitarbeiterverhalten die Marke stärkt, Wiesbaden, S. 4-33.

Kleemann, Frank (2012): Subjektivierung von Arbeit - Eine Reflexion zum Stand des Diskurses, in: Arbeits- und Industriesoziologische Studien 5, S. 6-20.

Kleemann, Frank / Christian Eismann / Tabea Beyreuther / Sabine Hornung / Katrin Duske / G. Günter Voß (2012): Unternehmen im Web 2.0. Zur strategischen Integration von Konsumentenleistungen durch Social Media, Frankfurt / Main - New York / NY.

Kleemann, Frank / Ingo Matuschek / G. Günter Voß (1999): Subjektivierung von Arbeit. Ein Überblick zum Stand der Diskussion (überarbeitete Fassung von: Zur Subjektivierung von Arbeit. Querschnittsgruppe Ökologie und Arbeit), Berlin, abrufbar unter: http://www.arbeitenundleben.de/downloads/ Subj_Klemann\%20 u.a.\%20SubjArb.pdf, letztes Abrufdatum: 9.8.2014.

Kleemann, Frank / Kerstin Rieder / G. Günter Voß (2008): Crowdsourcing und der Arbeitende Konsument, in: Arbeits- und Industriesoziologische Studien 1, S. 29-44.

Koch, Michael / Angelika C. Bullinger / Kathrin M. Möslein (2009): Social Software für Open Innovation. Die Integration interner und externer Innovatoren, in: Ansgar Zerfaß / Katrin M. Möslein (Hrsg.), Kommunikation als Erfolgsfaktor im Innovationsmanagement: Strategien im Zeitalter der Open Innovation, Wiesbaden, S. 159-176.

Kratzer, Nick (2003): Arbeitskraft in Entgrenzung. Grenzenlose Anforderungen, erweiterte Spielräume, begrenzte Ressourcen, Berlin.

Kratzer, Nick / Sarah Nies / Barbara Pangert / Gerlinde Vogl (2011): Leistungspolitik und Work-LifeBalance. Eine Trendanalyse des Projekts Lanceo, München, abrufbar unter: http://www.lanceo.de/ files/lanceo_brosch_re_lay_v3_downloadversion.pdf, letztes Abrufdatum: 12.8.2014.

Lohr, Karin (2003): Subjektivierung von Arbeit. Ausgangspunkt einer Neuorientierung der Industrieund Arbeitssoziologie?, in: Berliner Journal für Soziologie 4, S. 511-529. 
Lüdke, Hartmut (2000): Lebensstile und Konsum, in: Doris Rosenkranz / Norbert F. Schneider (Hrsg.), Konsum. Soziologische, ökonomische und psychologische Perspektiven, Leverkusen, S. 117-132.

Marschall, Jörg (2010): „So ein Auto ist eigentlich 'ne lebende Baustelle.“ Markengemeinschaften als Prosumentenkollektive, in: Birgit Blättel-Mink / Kai-Uwe Hellmann (Hrsg.), Prosumer Revisited. Zur Aktualität einer Debatte, Wiesbaden, S. 149-168.

Marx, Karl ([1867]1962): Das Kapital. Kritik der politischen Ökonomie, Buch 1: Der Produktionsprozess des Kapitals, Marx-Engels-Werke, Band 23, Berlin.

Mohe, Michael / Daniel Dormiok / Stephan Kaiser (2010): Auswirkungen von betrieblichen Work-LifeBalance Maßnahmen auf Unternehmen: Stand der empirischen Forschung, in: Zeitschrift für Management 5, S. 105-139.

Moldaschl, Manfred / G. Günter Voß (2003): Subjektivierung von Arbeit, 2. Auflage, München - Mering.

Moosbrugger, Jeanette (2012): Subjektivierung von Arbeit: Freiwillige Selbstausbeutung. Ein Erklärungsmodell für die Verausgabungsbereitschaft von Hochqualifizierten, 2. erweiterte Auflage, Wiesbaden.

Morhart, Felicitas M. / Walter Herzog / Torsten Tomczak (2009): Brand-specific leadership: turning employees into brand champions, in: Journal of Marketing 73, S. 122-142.

Müller-Neuhoff, Klaus / Wolfgang Giehl (2004): Fokus Internal Branding. Vom Mitarbeiter zum Mitmacher, Sternenfels.

Muster, Viola (2010): Companies promoting sustainable consumption of employees, in: Journal of Consumer Policy 34, S. 161-174.

Muster, Viola / Ulf Schrader (2011): Green Work-Life Balance: A new perspective for Green HRM, in: Zeitschrift für Personalforschung 25, S. 140-156.

Neyer, Ann-Katrin / Angelika C. Bullinger / Kathrin M. Möslein (2009): Integrating inside and outside innovators: a sociotechnical systems perspective, in: R\&D Management 39, S. 410-419.

Piller, Frank. T. / Dominik Walcher (2006): Toolkits for idea competitions: A novel method to integrate users in new product development, in: R\&D Management, 36, S. 307-318.

Pongratz, Hans J. / G. Günter Voß (2003): Arbeitskraftunternehmer - Erwerbsorientierungen in entgrenzten Arbeitsformen, Berlin.

Prahalad, C. K. / Venkat Ramaswamy (2000): Co-opting Customer Competence, in: Harvard Business Review 78, S. 79-88.

Reichwald, Ralf / Anton Meyer / Marc Engelmann / Dominik Walcher (2007): Der Kunde als Innovationspartner. Konsumenten integrieren, Flop-Raten reduzieren, Angebote verbessern, Wiesbaden.

Scherhorn, Gerhard (1977): Konsum, in: René König (Hrsg.), Band 11: Freizeit. Konsum, 2. Auflage, Stuttgart, S. 193-265.

Scherhorn, Gerhard (1980): Die Entstehung von Verbraucherproblemen im Spannungsfeld von Konsum und Arbeit, in: Journal of Consumer Policy 4, S. 102-114.

Schier, Michaela (2009): Räumliche Entgrenzung von Arbeit und Familie: Die Herstellung von Familie unter Bedingungen von Multilokalität, in: Informationen zur Raumentwicklung 1-2, S. 55-66.

Schmidt, Marc (2013): The integration of employees as consumers into innovation processes of sustainable products and services, Präsentation auf der 16. ERSCP Konferenz \& 7. EMSU Konferenz, 6. Juni 2013, Istanbul.

Schrader, Ulf / Frank-Martin Belz (2011): Nutzerintegration in Nachhaltigkeitsinnovationen: Ein- und Ausblicke, in: Frank-Martin Belz / Ulf Schrader / Marlen Arnold (Hrsg.), Nachhaltigkeitsinnovationen durch Nutzerintegration, Wirtschaftswissenschaftliche Nachhaltigkeitsforschung, Band 13, Marburg, S. 331-344. 
Schrader, Ulf / Christoph Harrach (2013): Empowering Responsible Consumers to be Sustainable Intrapreneurs, in: Ulf Schrader / Vera Fricke / Declan Doyle / Victoria W. Thoresen (Hrsg.), Enabling Responsible Living, Berlin - Heidelberg, S. 181-192.

Schrader, Ulf / Christoph Harrach / Laura Stanszus (2013): Ergebnisse einer Arbeitnehmerbefragung, abrufbar unter: http://nachhaltig-leben-und-arbeiten.de/umfrage, letztes Abrufdatum: 8.8.2014.

Schrage, Dominik (2009): Die Verfügbarkeit der Dinge. Eine historische Soziologie des Konsums, Frankfurt / Main - New York / NY.

Schweisfurth, Tim (2013): Embedded lead users inside the firm: How innovative user employees contribute to the corporate product innovation process, Wiesbaden.

Schweitzer, Rosemarie von (1991): Einführung in die Wirtschaftslehre des privaten Haushalts, Stuttgart.

Seebacher, Ulrike / Irmgard Schultz / Wilma Mert (2011): Nachhaltiges Handeln im beruflichen und privaten Alltag, in: Politische Ökologie 125, S. 114-115.

Shrum, L.J. / John McCarty / Tina M. Lowrey (1995): Buyer characteristics of the green consumer and their implications for advertising strategy, in: Journal of Advertising 24, S. 71-82.

Spreitzer, Gretchen M. (1995): Psychological empowerment in the workplace: Dimensions, measurement and validation, in: Acadamy of Management Journal 38, S. 1442-1465.

ThyssenKrupp AG (2011): 200 Jahre Krupp. Sonderveröffentlichung der ThyssenKrupp AG in Zusammenarbeit mit der WAZ Mediengruppe, abrufbar unter: http://www.derwesten.de/media/ 6076222--2893/200-Jahre-Krupp, letztes Abrufdatum: 20.8.2014.

Toffler, Alvin (1980): The third wave, New York / NY.

Voß, G. Günter (1991): Lebensführung als Arbeit. Über die Autonomie der Person im Alltag der Gesellschaft, Stuttgart.

Voß, G. Günter (1998): Die Entgrenzung von Arbeit und Arbeitskraft. Eine subjektorientierte Interpretation des Wandels der Arbeit, in: Mitteilungen aus der Arbeitsmarkt- und Berufsforschung 31, S. 473-487.

Voß, G. Günter / Hans J. Pongratz (1998): Der Arbeitskraftunternehmer. Eine neue Grundform der „Ware Arbeitskraft"?, in: Kölner Zeitschrift für Soziologie und Sozialpsychologie 50, S. 131-158.

Voß, G. Günter / Kerstin Rieder (2005): Der arbeitende Kunde. Wenn Konsumenten zu unbezahlten Mitarbeitern werden, Frankfurt / Main - New York / NY.

Wiswede, Günter (2000): Konsumsoziologie - Eine vergessene Disziplin, in: Doris Rosenkranz / Norbert F. Schneider (Hrsg.), Konsum. Soziologische, ökonomische und psychologische Perspektiven, Leverkusen, S. 23-72.

Zeppelin Wohlfahrt GmbH (2013): Historie der Zeppelin Wohlfahrt GmbH, abrufbar unter: http:// www.zeppelin-wohlfahrt.com/geschichte.html, letztes Abrufdatum: 12.8.2014.

Zwick, Detlef / Samuel K. Bonsu / Aron Darmody (2008): Putting consumers to work. ,Co-creation' and new marketing govern-mentality, in: Journal of Consumer Culture 8, S. 163-196.

Dipl. Soz.Wiss. Viola Muster

Technische Universität Berlin Institut für Berufliche Bildung und Arbeitslehre FG Arbeitslehre/ Ökonomie und Nachhaltiger Konsum

Marchstraße 23

10587 Berlin

viola.muster@tu-berlin.de 